\section{Contemporary dental practice in the UK: demographic details and practising arrangements in 2008}

\author{
P. A. Brunton, ${ }^{1}$ T. Burke, ${ }^{2}$ M. O. Sharif, ${ }^{3}$ E. K. Muirhead, ${ }^{4}$ S. Creanor ${ }^{5}$ \\ and N. H. F. Wilson ${ }^{6}$
}

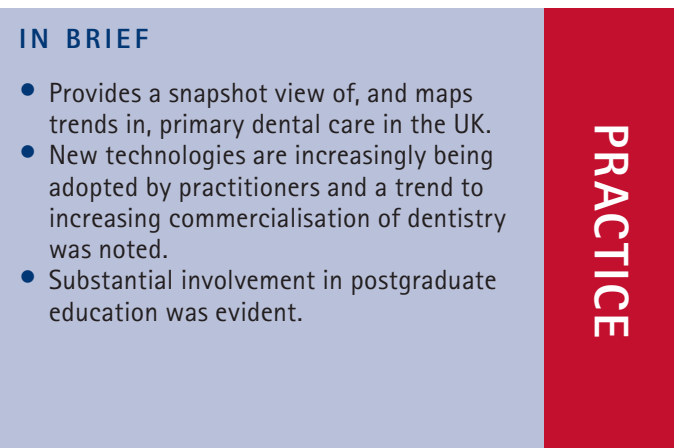

\begin{abstract}
Objectives To determine, by postal questionnaire, the demographic profile and practising details of general dental practitioners in the UK in 2008. Methods A piloted 89 question postal questionnaire was distributed in 2007/2008 to 1,000 dentists, with effective addresses in the UK, whose names and addresses were obtained by random selection from the General Dental Council (GDC) register. Results Six hundred and ninety-one questionnaires were returned, of which 662 were useable - an acceptable 66\% useable response rate. Of the respondents, 69\% were male and 59\% were practice principals. Fifty-three percent of the respondents' practices were in town or city centres with a wide geographic distribution. Single-handed practitioners accounted for $17 \%$ of respondents, with the mean number of dentists per practice being 3.6 (median 3.0). Typically, respondents' practices provided a mean of 26 patient care sessions per week, with each dentist treating on average 15 patients per session - 16 minutes per patient on average including surgery turnaround time, assuming 4 hour sessions. Hygienists typically treated seven patients per session - 34 minutes per patient on average including surgery turnaround time, assuming 4 hour sessions. Respondents stated that $57 \%$ of patients were treated under the NHS arrangements, with 28\% being private, $7 \%$ independent and $7 \%$ insurance-based. Responses indicated that $73 \%$ of the respondents used a computerised patient management system, 67\% had an internet connection and 60\% used email, principally for correspondence, ordering materials and other uses such as referrals and research. Forty-five percent of respondents owned an intra-oral camera, with $45 \%$ of those using it routinely. Regarding new concepts, the use of nickeltitanium endodontic files (61\%), digital imaging (28\%) and zirconia all-ceramic bridgework (27\%) were the most frequently cited innovations currently used by the respondents. Regarding the most notable changes in findings when compared with a related study conducted in 2000, these were connected to the method of payment with the proportion of NHS patients dropping to 57\% compared with 86\%; the volume of postgraduate education undertaken by dentists, with a 50\% increase in the proportion of respondents having attended five or more courses each year (63\% compared with 40\% in 2000); and the use of zirconia all-ceramic bridgework (27\% of respondents). Conclusions The findings of the present study are considered to indicate increasing commercialism of dentistry in the UK, with evidence of many practitioners adopting new technologies, underpinned by substantial participation in postgraduate education.
\end{abstract}

\section{INTRODUCTION}

Patterns of dental disease have changed dramatically over the past quarter of a century, with the resultant patient benefit

"Restorative dentistry, Leeds Dental Institute, Clarendon Way, Leeds LS2 9LU; ${ }^{2}$ University of Birmingham, School of Dentistry, St Chad's Queensway, Birmingham B4 6NN; ${ }^{3}$ University of Manchester, School of Dentistry, Oral Health Unit, Higher Cambridge Street, Manchester M15 6LP: ${ }^{4}$ Primary Dental Care, University of Birmingham, Birmingham B4 6NN; ${ }^{5}$ Biostatistics and Epidemiology Group, University of Plymouth, Plymouth PL6 8BX; ${ }^{6}$ Restorative dentistry, GKT Dental Institute,

Kings College University London, London SE1 9RT

${ }^{*}$ Correspondence to: Paul Brunton

Email: p.a.brunton@leeds.ac.uk; Tel: 01133436182

\section{Refereed Paper}

Accepted 28 October 2011

DOI: 10.1038/sj.bdj.2011.1098

${ }^{\circ}$ British Dental Journal 2012; 212: 11-15 being realised in increased numbers of teeth retained, lower levels of edentulousness and higher patient expectations, albeit only in some members of UK society. ${ }^{1}$ The impact of increasing media coverage of oral and dental issues, including so-called 'makeover' shows, has increased public awareness of the benefits of oral health and dental attractiveness; although the disadvantages of certain interventive techniques, in particular the long-term consequences, do not appear to receive sufficient attention. ${ }^{2}$ The increasing commercialisation of the dental profession is reflected in a number of ways, including the burgeoning numbers of courses on the business aspects of dentistry and the marketing of alternative forms of treatment. Against this background, it was considered timely to investigate the practising arrangements of UK dentists in $2000 .^{3-5}$ Given the ever-increasing rate of change impacting on the clinical practise of dentistry, it was considered appropriate to repeat the investigation six years later.

It is therefore the purpose of the present paper to report the results of a mailed survey of a sample of UK dentists in respect of their demographic profile and practising arrangements. A further aim was to compare the results of this survey with the previous survey to investigate changes in practice demographics and arrangements over the elapsed 4 year period. Two other 
papers report the results in relation to the materials and techniques used for direct and indirect restorations and related matters.

\section{METHODS}

A questionnaire, based upon the previous questionnaire that was successfully used for the earlier survey, ${ }^{3}$ was piloted among ten practitioners in NW England. To facilitate comparison with the results from the earlier study, no questions were changed other than those pertaining to hardware and IT capability, which required updating. The questionnaire therefore employed a total of 89 questions, many of which comprised a number of elements.

The questionnaire was distributed by post, with a covering letter and stamped, addressed envelope, to a group of 1,000 primary dental care practitioners based in the UK and selected at random from the Dentist Register. The selection procedure aimed to include a wide geographic distribution of practitioners across the UK. After four weeks, a second copy of the questionnaire was sent, together with a covering letter, to non-responding practitioners.

The data from the returned questionnaires were analysed using Minitab (version 15, Minitab Inc.) and StatXact (version 8, Cytel Inc.). Summary statistics (means and standard deviations [SD], medians and ranges) are presented as appropriate to each question. Percentages given are based upon the number of responses for the question of interest, given that not all respondents answered all the questions. Cross tabulations and chi-squared tests, as well as the two-sample t-test, Mann Whitney test and Kruskal Wallis test were used to assess significant relationships between a number of the demographic variables and other questions of interest, as appropriate. The level of significance was set at $1 \%$ because of the number of statistical tests being carried out, in order to reduce the chance of getting false positive results.

\section{RESULTS}

\section{Demographic data}

A total of 691 questionnaires were returned, of which 662 were useable, giving an effective response rate of 66\%.

of the respondents, 67\% ( $\mathrm{n}=441)$ were male, $53 \%(\mathrm{n}=336)$ were practice principals, $42 \%(\mathrm{n}=264)$ associates,

\section{Table 1 Findings in respect of participation in postgraduate education}

\begin{tabular}{|l|l|l|}
\hline Number of courses & Percentage of respondents & $\begin{array}{l}\text { Number of respondents } \\
\text { (total of } 661 \text { responses) }\end{array}$ \\
\hline 0 & 2 & 12 \\
\hline $1-2$ & 11 & 74 \\
\hline $3-4$ & 24 & 161 \\
\hline 5 or more & 63 & 414 \\
\hline
\end{tabular}

\begin{tabular}{|c|c|c|}
\hline Type of camera & Percentage of respondents & $\begin{array}{l}\text { Number of respondents } \\
\text { (total of } 295 \text { responses) }\end{array}$ \\
\hline $35 \mathrm{~mm}$ & 17 & 48 \\
\hline Digital & 16 & 186 \\
\hline Video & 17 & 49 \\
\hline
\end{tabular}

\section{Table 3 Findings in respect of lasers}

\begin{tabular}{|l|l|l|}
\hline Comment & $\begin{array}{l}\text { Percentage of } \\
\text { respondents }\end{array}$ & $\begin{array}{l}\text { Number of respondents } \\
\text { (total of 649 responses) }\end{array}$ \\
\hline I do not own a laser and would not like to & 66 & 431 \\
\hline I do not own a laser but would like to & 31 & 202 \\
\hline I own a laser and use it & 2 & 14 \\
\hline I own a laser and do not use it & $<1$ & 2 \\
\hline
\end{tabular}

Table 4 Findings in respect of glove use

\begin{tabular}{|l|l|l}
\hline Type of glove & Percentage of respondents & $\begin{array}{l}\text { Number of respondents } \\
\text { (total of 644 responses) }\end{array}$ \\
\hline Powdered latex & 11 & 68 \\
\hline Powdered latex-free & 3 & 17 \\
\hline Powder-free latex & 71 & 456 \\
\hline Powder-free latex-free & 16 & 103
\end{tabular}

$2 \%(\mathrm{n}=14)$ assistants and 3\% $(\mathrm{n}=20)$ vocational trainees.

The respondents' mean number of years since graduation was 18.2 (median 18.5, range 0 to 53). There was evidence of a significant difference between the average number of years since graduation of female dentists compared to male dentists, with female respondents graduating more recently by an average of 5 years $(\mathrm{p}<0.001)$.

The majority of respondents (83\%; $n=533 / 644$ ) worked in partnership/group practice arrangements, with single-handed practitioners accounting for the remaining $17 \%(n=111 / 644)$ of respondents. The mean number of dentists per practice was found to be 3.6 (SD 2.1, median 3.0, range 1 to 14 ) and the mean number of hygienists per practice was 1.1 (SD 1.1, median 1.0 , range 0 to 7 ). The mean number of qualified dental nurses per practice was reported to be 3.8 (SD 3.2, median 3.0, range 0 to 40 ), while the mean reported number of unqualified dental nurses per practice was 1.7 (SD 1.9, median 1.0, range 0 to 15). The data did not include any evidence of practitioners working without the support of a dental nurse.

Regarding practice location, 53\% ( $n=345 / 650)$ of practices were in town or city centre locations, 34\% $(n=218 / 650)$ were in a suburban location, and 13\% ( $n=87 / 650$ ) were in a rural location. 


\section{Method of patient payment}

Respondents were asked the question: "what proportion of your patients are NHS independent, insurance based, private, or "other" funding arrangement?' The responses $(n=654)$ indicated that $57 \%$ of patients were treated under the NHS arrangements, with 28\% being treated under private arrangements, 7\% independent, 6\% insurance-based and 1\% under 'other' arrangements, with 'other' being found to include Armed Forces ( $\mathrm{n}=5)$, and community $(\mathrm{n}=3)$.

\section{Postgraduate education}

Regarding the respondents' reported participation in postgraduate education, findings are shown in Table 1.

There was no evidence of a significant association between the number of postgraduate courses attended and whether the respondent worked in a mainly NHS practice or not $(\mathrm{p}=0.533)$.

\section{Practice work pattern}

Typically, respondents' practices provided a mean of 26 patient care sessions per week (SD 18.4, median 22, range 1 to 200), with each dentist treating a mean of 15 patients per session (SD 7.4, median 15, range 0 to 76 ) - that equates to 16 minutes per patient, including surgery turnaround time, assuming four hour sessions with no breaks.

Hygienists were reported typically to treat seven patients per session (median 8 , range 0 to 38) - 34 minutes per patient, including surgery turnaround time, assuming four hour sessions with no breaks.

There was, on average, a significantly greater number of patients per session in mainly NHS practices compared to other practices (medians of 25 (range 2 to 200) and 18 (range 1 to 100), respectively; $\mathrm{p}<0.0001$ ). Similarly, on average, a greater number of patients were treated per dentist per session in mainly NHS practices compared to other practices (medians of 14 (range 5 to 76) and 10 (range 0 to 55), respectively; $p<0.0001)$. There was insufficient evidence to conclude a significant difference between mainly NHS and mainly other practices in terms of the average number of patients treated by each hygienist per session (medians of 7 (range 0 to 30 ) and 8 (0 to 30), respectively; $p=0.002$ ).

\section{Use of practice-based computers}

Responses indicated that $73 \%$ of respondents ( $\mathrm{n}=479 / 661$ ) used a computerised patient management system, 67\% ( $\mathrm{n}=440 / 662$ ) had an internet connection and 60\% ( $\mathrm{n}=392 / 657$ ) used email, principally for correspondence ( $87 \% ; n=341 / 392)$, ordering materials ( $42 \% ; n=166 / 392)$ and other uses (13\%; $n=50 / 392)$ such as, referrals, communication, job applications and research into products.

Respondents who had a computerised patient management system had on average graduated more recently than respondents who did not have a computerised patient management system, by between 1.4 and 5.5 years $(p=0.001)$.

There was no evidence of a difference in average years since graduation between (1) those who did and did not have an internet connection ( $p=0.265)$, nor (2) respondents who did or did not use email $(p=0.822)$.

Regarding transmission of payment claim forms, 29\% ( $\mathrm{n}=186 / 646)$ of respondents stated that they never used this, $57 \%(\mathrm{n}=366 / 646)$ routinely used this, with $13 \%(n=83 / 646)$ reporting that they did not work in the NHS and, as such, did not have need for such a facility.

\section{Innovations}

The data collected indicated that 45\% ( $n=295 / 656)$ of respondents owned an intra-oral camera, with 48\% ( $\mathrm{n}=142 / 295)$ using it routinely and 44\% ( $\mathrm{n}=130 / 295)$ occasionally. The type of camera used is detailed in Table 2.

Concerning recently introduced concepts and techniques, the use of nickel-titanium endodontic files (61\%; $n=404 / 662$ ), digital radiography/imaging (28\%; $\mathrm{n}=182 / 662)$, zirconia all-ceramic bridgework (27\%; $\mathrm{n}=178 / 662)$, fibre-reinforced resin composite bridgework $(15 \% ; n=101 / 662)$ and air abrasion tooth preparation (13\%; $\mathrm{n}=86 / 662$ ) were the most frequently cited innovations adopted by the respondents.

Regarding implant dentistry, $80 \%$ of respondents $(n=526 / 654)$ stated that they did not place or restore them. One percent ( $n=7 / 654$ ) carried out implant surgery only, $8 \%(n=51 / 654)$ undertook implant prosthodontics and 11\% ( $=70 / 654)$ carried out both implant surgery and prosthodontics.

Regarding the use of lasers, the results are presented in Table 3.
Regarding the provision of orthodontic treatment, $70 \%$ of the respondents ( $n=458 / 655)$ stated that they 'never' provided this, while $4 \%(\mathrm{n}=155 / 655)$ provided orthodontics 'occasionally' and 7\% ( $\mathrm{n}=43 / 655$ ) 'routinely'.

\section{Preventive dentistry}

Regarding their use of fluoride, 20.4\% ( $n=135)$ of respondents stated that they did not use topical fluoride. However, $39.9 \%(n=264)$ used practice-based fluoride gel treatment, and 6.6\% $(n=44)$ used practice-based liquid fluoride mouth rinses. Regarding the use of fluoride gel for patients' home use, 30.8\% ( $\mathrm{n}=204)$ stated that they prescribed this, while $64.4 \%$ ( $n=426$ ) prescribed a fluoride mouth rinse.

Regarding their prescription of fissure sealant, 43.4\% ( $\mathrm{n}=284)$ of respondents stated that they used these 'routinely', $51.6 \%(\mathrm{n}=344)$ 'occasionally' and 4\% ( $\mathrm{n}=26$ ) 'never'.

\section{Equipment}

When asked about their preferred style of delivery of chair-side equipment, $62 \%$ of respondents $(n=403 / 655)$ stated 'cart', while 30\% ( $\mathrm{n}=199 / 655)$ stated 'over the patient', and the remaining $8 \%(\mathrm{n}=53 / 655)$ a variety of methods. Regarding contamination of compressed air, 47\% of respondents ( $\mathrm{n}=313 / 662$ ) stated that they did not note contamination, while $8 \%(n=51 / 662)$ noted oil contamination and 19\% (n = 125/662) water contamination.

\section{Pain and anxiety control}

Local anaesthesia was the most commonly employed method of pain control, with $97 \%$ of respondents ( $n=645 / 662$ ) using this. Intravenous sedation was applied by $20 \%$ ( $n=130 / 662)$, nitrous oxide by $13 \%$ ( $\mathrm{n}=89 / 662)$, acupuncture by $4 \%$ ( $n=26 / 662)$, hypnosis by $3 \%(n=20 / 662)$ and TENS (transcutaneous electrical nerve stimulation) by $0.5 \%(\mathrm{n}=3 / 662)$. Thirtytwo percent of respondents $(n=214 / 662)$ referred patients for treatment under general anaesthetic.

\section{Infection control}

Gloves were reportedly worn for operative procedures by $99 \%$ of respondents ( $n=647 / 657$ ), with the preferred types of glove being shown in Table 4 . 
Regarding sterilisation of handpieces, $98 \%$ of respondents $(n=644 / 658)$ indicated that they sterilised their high-speed handpieces after every patient use, the equivalent figures for slow handpieces being 97\% ( $n=640 / 660)$. A private repair service was used by $61 \%$ of respondents ( $\mathrm{n}=390 / 642$ ) while $35 \%$ of respondents ( $n=223 / 642$ ) relied on the manufacturer of their handpieces.

Regarding three-in-one syringes, 64\% of respondents ( $\mathrm{n}=422 / 659)$ used disposable three-in-one syringes, and of the remainder who did not ( $\mathrm{n}=237), 98 \%$ ( $\mathrm{n}=231)$ used metal tips autoclaved between patients.

\section{Health of dentists}

Regarding the responses to the questions in respect of health, there were 660 responses. The mean reported number of days in the previous year for which respondents were absent from work because of illness was 4 (SD 18.5, median 1, range 0 to 365). No illness predominated in the responses, but reasons for absence included bereavement, urinary tract infection, chronic back problems, maternity leave and trauma, including skiing accidents.

There was insufficient evidence to discern a difference between mainly NHS and other dentists, in terms of number of sick days off ( $p=0.087)$.

\section{DISCUSSION}

The present study sought to investigate aspects of the demographic of general dental practice in the UK in 2008. This was considered relevant subsequent to a previous study ${ }^{3-5}$ and an ever-increasing rate of change in circumstances considered to impact on the clinical practice of dentistry. Information of the type revealed by the present investigation is considered important to understanding, among other factors, trends in oral health provision, attitudes in the profession and future requirements in terms of education and recruitment to studies leading to membership of the dental team.

The study used a postal questionnaire. This approach could be considered to have achieved a good response at 66\%, especially when the length of the questionnaire is taken into account, given the mean response rate of postal questionnaires to dentists has previously been reported to be $64 \%$ (range $17 \%$ to $100 \%)^{6}$ and other means of collecting the range of information being sought have not been validated. The dentists to whom the questionnaire was distributed had a wide geographic distribution, with responses having been received from all parts of the UK. Despite the relatively large response and the favourable geographic distribution, it cannot be claimed that the findings are representative of all areas of the UK, let alone all practitioners; however, it is suggested that the findings, despite their possible limitations, shed important light on general dental practice in the UK in 2008.

\section{Trends since 2000}

The questionnaire was adapted in only two sections from the one used in a related survey in $2000 .^{3}$ In this way, being mindful of differences in sampling and the geographic distribution of response, certain comparisons to the fundings were considered relevant in terms of identifying general trends.

\section{Demographics}

The proportion of respondents who were male in 2000 was $73 \%$. In the present study this had dropped to 67\% in keeping with the increasing 'feminisation' of the profession. The number of practice principals also dropped from 65\% in 2002 to 53\% in 2008. Speculation as to whether 'feminisation' of the profession is associated with an apparent reduction in practice principals would be conjecture. Additionally, the modest increase in the proportion of practitioners in a town or city centre location from $49 \%$ in 2002 to $53 \%$ in 2008 could be viewed as important, but this would need to be the subject of further research to confirm any trend in the geographic distribution of dental practices.

\section{Methods of payment}

of particular note is the comparison in methods of payment for dentistry. When asked the question 'what proportion of your patients are NHS?', the response in 2002 was 86\%. In 2008, this figure had dropped to 57\%. A corresponding rise in provision of private care was apparent, from $9 \%$ in 2002 to $28 \%$ in the present study, while insurance-based payment rose from $2 \%$ in 2002 to $7 \%$ in 2008 . This is viewed as significant in terms of the increasing proportion of the population availing themselves of dental services, given the efforts made to allow increasing access of individuals to NHS dental services, which according to the present findings only contribute to 57\% of practitioners' patient base.

\section{Postgraduate education}

There would appear to have been an overall increase in the volume of postgraduate education undertaken by dentists from 2002 to 2008; specifically with 63\% of the respondents to the present study stating that they attended five or more courses per year, compared with $41 \%$ in 2002 . Notwithstanding the introduction of compulsory continuing professional development by the General Dental Council, the apparent substantial increase in engagement in postgraduate education, which would appear to greatly exceed statutory requirements, could be considered to reflect increasing awareness of the need to keep abreast of innovations in clinical practice.

\section{Use of computers}

As might be expected, given the global increase in the use of computers, the respondents' use of computer technology has increased since 2002, with internet connection increasing from 31\% in 2002 to $67 \%$ in 2008 . No comparison is possible with regards to the use of a computer management system or use of email, as questions in respect of these aspects of clinical practice were not included in the previous study. The use of email by dental practitioners at $60 \%$ of respondents, could, however, be considered low in comparison to other professions and sections of society.

\section{Cameras and magnification}

The proportion of respondents who used an intra-oral camera regularly to support their clinical practice increased from 21\% in 2002 to $45 \%$ in the present study, with 'digital' showing a large increase, from $26 \%$ in 2002 to $66 \%$ in 2008. The latter data may simply reflect what is happening in the world of photography in general. However, the increased use of intra-oral photography could be viewed as encouraging in terms of record keeping, communications with patients, and possibly communications between practitioners and dental technicians in the prescription of indirect restorations. 


\section{Pain and anxiety control}

Local anaesthesia remained the most commonly employed method of pain control. There were only minor changes in other aspects of pain and anxiety control, with the exception of the proportion of respondents continuing to refer patients for treatment under general anaesthesia which reduced from 56\% in 2002 to 32\% in the present study. Whether this reflects a decrease in the number of extractions required for, in particular, children, or that increasing numbers of teeth are being extracted under local rather than general anaesthesia is not known. One way or another, a reduced reliance on the use of general anaesthesia is to be welcomed.

\section{Infection control}

Gloves were reportedly worn for operative procedures by $99 \%$ of respondents, an increased proportion of respondents when compared with the 2002 figure of 92\%. While this represents a much-needed improvement, there remains an anxiety that some respondents, albeit 1-2\%, did not feel that it was appropriate to wear gloves routinely, given the potential for infection between patients or from operator to patient when gloves are not worn ${ }^{7}$ and the accepted concept of universal precautions. ${ }^{8}$ There was a notable trend away from powdered latex gloves, from 33\% of respondents in 2002 , to $11 \%$ in 2008 ; a concurrent increase in the wearing of powder-free gloves, from 54\% of respondents in 2002 , to $71 \%$ in 2008 ; and an increase from $7 \%$ powder-free latex-free in 2002 to $16 \%$ of this glove type in 2008 .

\section{Health of dentists}

Regarding days absent from work, the finding of four days on average per annum was similar to that in 2002. This low rate of absenteeism is considered to indicate that levels of ill-health among general dental practitioners have not changed in recent years.

\section{Innovations}

Regarding the adoption of innovations, the use of zirconia all-ceramic bridgework (27\% of respondents) may be considered the most surprising, given that zirconia systems were not commercially available at the time of the 2002 survey. This could be considered to reflect patient wishes for metal-free restorations, together with the clinicians' desire for restorations which contain no substances which are potential allergens and provoke an unfavourable tissue reaction. This could also reflect the growing awareness of the potential success of zirconia frameworks in bridges and crowns. ${ }^{9}$ No previous figures are available for practitioner provision of implant dentistry. The involvement of $21 \%$ of respondents in one or more aspects of implant therapy is an indication of the impact of this treatment modality on oral healthcare in the UK. Implant dentistry, together with the use of novel systems, including zirconia allceramic bridgework, is understood to occur under primarily private contract in general dental practice. The evidencebase for in particular implant dentistry has, however, developed to the extent that at least simpler forms of implant treatment should, through appropriate education and training, become part of mainstream clinical practice.

Subsequent papers in this series will report findings in respect of materials and techniques used in general dental practice for direct restorations, endodontics and bleaching as well as indirect restorations and fixed prosthodontics.

\section{CONCLUSION}

The findings of the present study are considered to give an indication of increasing commercialisation of dentistry in the UK, with evidence of many practitioners adopting new technologies, underpinned by substantial participation in postgraduate education. While apparent trends in infection control are encouraging, the adoption of even basic forms of information technology has been found to be relatively low among dental practitioners in the UK.

Many thanks are due to the dentists who took the time and trouble to complete and return their questionnaire.

1. Department of Health. Adult dental health survey: oral health in the United Kingdom 1998. London: The Stationery Office, 2000.

2. Burke F J, Kelleher M G. The 'daughter' test in elective esthetic dentistry. J Esthet Restor Dent 2009; 21: 143-145.

3. Burke F J, Wilson N H, Christensen G J, Cheung S W, Brunton P A. Contemporary dental practice in the UK: demographic data and practising arrangements. Br Dent J 2005; 198: 39-43.

4. Brunton PA, Christensen G J, Cheung S W, Burke $\mathrm{FJ}$, Wilson $\mathrm{N} \mathrm{H}$. Contemporary dental practice in the UK: indirect restorations and fixed prosthodontics. Br Dent J 2005; 198: 99-103.

5. Wilson N H, Christensen G J, Cheung S W, Burke F J, Brunton P A. Contemporary dental practice in the UK: aspects of direct restorations, endodontics and bleaching. Br Dent J 2004; 197: 753-756.

6. Tan R T Burke FJ. Response rates to questionnaires mailed to dentist. A review of 77 publications. Int Dent J 1997; 47: 349-354.

7. Allen A L, Organ R J. Occult blood contamination under the fingernails: a mechanism for the spread of blood-borne infection. J Am Dent Assoc 1982; 105: 455-459.

8. Centre for Disease Control. Recommendations for preventing transmission of human immunodeficiency virus and hepatitis $B$ virus to patients during exposure-prone invasive procedures. MMWR Recomm Rep 1991; 40(RR-8): 1-9.

9. Crisp R J, Cowan A J, Lamb J, Thompson O, Tulloch $\mathrm{N}$, Burke F J. A clinical evaluation of all-ceramic bridges placed in patients attending UK general dental practices: three-year results. Br Dent J 2008; 205: 477-482. 